\title{
Stellar Structure.
}

THE investigation of the structure of the stars, which has for long been a subject of disagreement between Sir James Jeans and Sir Arthur Eddington, has now entered on a new phase through the work of Prof. E. A. Milne. A long paper on this matter by Prof. Milne, which appears in the November number of the Monthly Notices of the Royal Astronomical Society together with related papers by Messrs. R. H. Fowler, N. Fairclough, and T. G. Cowling, was introduced to the Society in November last, but no time was available for discussion. The whole of the meeting of the Royal Astronomical Society on Friday, Tan. 9, was accordingly devoted to a debate on the subject, which was briefly opened by Prof. Milne, and in which many astronomers and mathematicians took part.

Prof. Milne's vicws were outlined in NATURE of Jan. 3, p. 16. In opening the discussion, he stated that the pioneer work in the subject had been done by Sir Arthur Eddington, and what he himself had done was to rationalise Eddington's theory by clearing it of ad hoc hypotheses. He could not accept Sir James Jeans's theory, because it depended on unlikely extrapolations of the laws of physics. He considered that the mass-luminosity law which Eddington claimed to have established was not a possible deduction from the fundamental formulæ. It could follow only from the addition of a missing equation expressing the unknown dependence on physical conditions of the rate of generation of energy in stars. He had, therefore, begun his investigations with observed quantities, representing conditions at the surface of the star, and had worked inwards, without making any assumptions unwarranted by observation.

Sir Arthur Eddington, who followed, pointed out that disagreements in physical discussions could be of two kinds : first, those which depended on the adoption of different assumptions or hypotheses in the absence of definite knowledge; and secondly, disagreements on the logical or mathematical deductions from given premises. He thought that the disagreement between Sir James Jeans and himself was entirely of the first kind; but between Prof. Milne and himself there appeared to be a mathematical disagreement, which was very unfortunate, He did not approach Prof. Milne's latest work in a spirit of opposition. He regarded it as quite a permissible attempt to improve the existing theory in points where room for improvement undoubtedly existed. The main feature of Milne's picture of the stars was that the mass was largely concentrated in a dense hot core with a surrounding rarefied. envelope. His own theory gave a much more gradual and uniform increase of density from the centre outwards. For the sun it gave a central density of 70, whereas Milne's theory gave a value of about 700,000 . The matter there was therefore, aceording to Milne, not in the condition of a perfect gas, and hence the mass-Iuminosity relation could not be deduced. There were certainly loopholes in that relation-it did not apply to a star composed mainly of hydrogen, for example-but he did not believe that Milne's theory had sealed up those loopholes. Milne himself had confessedly not been able to satisfy himself that the equations for his stars possessed solutions, and Sir Arthur thought that it was a priori unlikely that they did. If such solutions existed, however, it still remained to decide between the two widely different central densities. This might be done by considering the intrinsic opacity in the interior, or its average if it was variable. An appeal on this ground, however, would show that both theories were wrong-that was the long-outstanding discrepancy between the physical and astronomical opacities of matter. Both theories required a larger opacity than would follow from current physical theory, but he had found that the discrep. ancy on Milne's theory was far greater than that on his own. A great deal depended on how large a mass was concentrated at the centre.' If it was merely a point-source of extremely high temperature and density, with the rest of the star following a distribution of density almost identical with that of his own theory, he might be willing to accept the modification as a useful addition to that theory : it would provide a source of stellar energy. The large amount of mass, however, which Milne placed around the centre, inevitably made worse the existing discrepancy with regard to the opacity. Prof. Milne had objected to his theory being called a hypothesis, regarding it rather as an inevitable conclusion. It was inevitable only if the premises were granted, and Sir Arthur had been denying them for fourteen years.

Sir James Jeans found himself in almost complete agreement with what Sir Arthur Eddington had said, hecause Sir Arthur had not touched on the points on which they differed. If Sir Arthur, Prof. Milne, and himself all suddenly became infallible and omnipotent as mathematicians, he and Sir Arthur would still differ on the same points as before, but Prof. Milne would agree with one or the other accordingly as he considered the stars to be gaseous inside or otherwise. There was nothing new in Milne's theory; all that was accurate in it had been done previously by Eddington or himself. He had shown long ago that if you integrated outwards from the centre of a star you got definite results right up to the boundary, but that there an infinite number of solutions were possible, and Nature itself decided which was the actual one. When, therefore, Milne reversed the process by integrating from the boundary inwards, it did not matter with which of those solutions his initial data corresponded; he would necessarily arrive at the same conditions in the interior. The work, however, would be much more cumbersome. $\mathrm{He}$ agreed with Eddington about the opacity discrepancy, but considered that the case had not been put strongly enough. The factor 10 in the luminosity, by which Eddington's own theory deviated from observation, was much greater than was permissible. Expressed in terms of volume, it meant that the sun should be as big

No. 3195, VoL. 127] 
as Antares. Since Milne's theory made matters worse still, he considered that that theory was ruled out of court entirely.

Dr. W. M. Smart directed attention to the fact that the problem was an idealised one, since we knew nothing at all about the interior of a star from observation. Assumptions had to be made, and it should not be forgotten that they were assumptions. He considered the stars themselves were the final umpires in the matter. Sir Arthur Eddington had suggested the opacity as the criterion by which the umpires gave their decision; he would suggest one of several other criteria. Sir James Jeans's theory had been very successful in explaining the formation of spectroscopic binaries by fission, but he did not think that Prof. Milne's centrally condensed stars would provide a satisfactory explanation.

Prof. F. A. Lindemann hoped that Prof. Milne would maintain his theory, because it was the only one which gave really high temperatures in the stars. The temperatures required by Sir Arthur Eddington were, on thermodynamical grounds, incapable of permitting the conversion of matter into radiation. It was all very well to say that Milne's conclusions were inevitable only if you presupposed something. Something had to be presupposed, and Eddington's ad hoc assumptions did not meet the facts of the generation of energy. He considered that the discrepancy of a million-fold in the densities of stars according to Eddington's theory was sufficiently large to make the increase to several million-fold in Milne's theory of no significance in deciding between the theories.

Sir Oliver Lodge remarked that what struck him most in the discussion was the remarkable agreement between the three protagonists. They were agreed on knowledge of fundamental importance which was not available twenty years ago, and, in comparison with that, the differences were unimportant. His idea of dealing with the problem would be to start with what we know-the surface temperature of the sun, its rate of generation of energy, the fact that energy comes from the disintegration of atoms. Prof. Lindemann had assured us that the last-named process required a very high temperature. Let that temperature at the centre be assumed. Then we knew the relation between radiation pressure and gravitational pressure, and with regard to the opacity the Compton effect would give us some information. From all these data a mathematician might work out some definite result. He was fascinated by Milne's theory of a nova resulting from the collapse of a star. Such a possibility had not occurred to him, but it seemed to work out.

Several other speakers contributed to the discussion, which concluded with brief comments by Prof. Milne. He did not agree that his work covered the same ground as Sir James Jeans's. He had started from the surface and worked inwards because it was only the surface that we could observe, and he had avoided assumptions which were necessary if you started in an unknown region.

Although it could not be said that the discussion led to a greater measure of agreement between the various speakers, it undoubtedly helped, by bringing together different methods of dealing with the question, to focus the nature of the problem more clearly in the minds of those present.

\section{Vitamin B.}

Vitamins $B_{2}$ AND $B_{3}$ : Bros.

THE possibility of obtaining vitamin $B_{1}$ in a 1 relatively pure condition has facilitated the differentiation of the other factors which, with $B_{1}$, make up the vitamin $B$ complex. Chick and Roscoe used Peters' concentrate to demonstrate that the rat required two factors, the second being known as $B_{2}$ or the antipellagrous vitamin. More recently, they have published papers dealing with the chemical properties of this factor (Biochem. Jour., vol. 23, pp. 504 and $514 ; 1929$ : vol. 24, p. $105 ; 1930$ ).

Yeast extracts contain vitamin $\mathrm{B}_{2}$, but the final antineuritic concentrate none : examination of the by-products of the concentration showed that about half the $B_{2}$ was precipitated by lead acetate, in the treatment of the extract with this reagent (at $p \mathrm{H} 4.5$ ) and another third in the treatment with baryta and sulphuric acid, the remainder being precipitated during the treatment with acid mercuric sulphate and the subsequent passage of hydrogen sulphide through the filtrate. The lead acetate precipitate was the most convenient source for obtaining a concentrated preparation: examination of this stage in detail showed that all the vitamin was carried down when the precipitation was carried out at a neutral reaction, but less than half at $p \mathrm{H} 2 \cdot 6$. The vitamin was recovered by decomposing the precipitate with hydrogen sulphide: to ensure precipitation of the lead sulphide at an acid reaction, at which the vitamin is not adsorbed on the precipitate, it was necessary first to hydrolyse the yeast gum in the extract with hydrochloric acid. Unfortunately, the lead precipitate also carries down some vitamin $B_{1}$, and it was not found possible to obtain a preparation of $B_{2}$ free from $B_{1}$ by dialysis, by making use of their different solubilities in alcohol or their different rates of destruction by ultra-violet light. The concentrate was active in a dose of $0.03 \mathrm{gm}$., equivalent to $0.5 \mathrm{gm}$. dried yeast daily. It is possible that yeast extract is an unsuitable medium for effecting a separation. Rosedale (ibid., vol. 21, p. 1266; 1927) precipítated from rice polishings extract (by means of lead acetate) a factor which was not $\mathrm{B}_{1}$, although enabling pigeons to grow and maintain health on a diet of polished rice. It cannot yet be said with certainty, however, that this factor is vitamin $B_{2}$.

B. T. Narayanan and J. C, Drummond have also carried out experiments on the concentration of vitamin $\mathrm{B}_{2}$ (ibid., vol. 24, p. $19 ; 1930$ ). Yeast

No. 3195, VoL. 127] 